\title{
Electron-Phonon Correlation Effects in Molecular Transistors
}

\author{
C. A. Balseiro, ${ }^{1}$ P. S. Cornaglia, ${ }^{2}$ and D. R. Grempel ${ }^{* 3}$ \\ ${ }^{1}$ Instituto Balseiro and Centro Atómico Bariloche, \\ Comisión Nacional de Energía Atómica, 8400 Bariloche, Argentina. \\ ${ }^{2}$ Centre de Physique Théorique, École Polytechnique, CNRS, 91128 Palaiseau Cedex, France. \\ ${ }^{3}$ CEA-Saclay, DSM/DRECAM/SPCSI, Bât. 462, F91191 Gif sur Yvette, France.
}

(Dated: June 20, 2017)

\begin{abstract}
The interplay of electron-electron and electron-phonon interactions is studied analytically in the Kondo regime. A Holstein electron-phonon coupling is shown to produce a weakening of the gate voltage dependence of the Kondo temperature and may explain the observed anomalies in some of these devices. A molecular center-of-mass mode opens a new channel for charge and spin fluctuations and in the antiadabatic limit the latter are described by an asymmetric two-channel Kondo model. Below the Kondo temperature the system develops a dynamical Jahn-Teller distortion and a low energy peak emerges in the phonon spectral density that could be observed in Raman microscopy experiments.
\end{abstract}

PACS numbers: 72.15.Qm, 73.22.-f

\section{INTRODUCTION}

One of the most exciting aspects in the physics of molecular transistors is the interplay between electronelectron and electron-phonon (e-ph) interactions. 1 Molecules attached to leads act like small quantum dots with broadened but well defined electronic levels and large charging energies. Electron-electron interactions lead to the well known Coulomb blockade effect 2,3 and the strongly correlated Kondo physics, 2,4,5,6 When the coupling of electronic degrees of freedom and molecular vibrational modes is strong, a rich variety of behavior emerges. $1,2,3,4,5,6,7,8,9,10,11,12,13,14,15,16,17,18,19$ The presence of steps in the current-voltage characteristics of molecular transistors can be explained by the coupling to a well defined phononic mode. Other features, like the anomalous behavior of the Kondo temperature as a function of the gate voltage, remain yet to be understood. $\underline{4.6}$ Center-of-mass oscillations of the active component of a transistor and electron-electron interactions are expected to produce a shuttling effect in nanomechanical devices ${ }^{20}$ The interplay of such mode with strong correlations at low temperatures is quite a complicated problem of general interest and experimental relevance..

Here we consider a molecule in the Kondo regime with a Holstein coupling and a center-of-mass motion mode (CMM) that modulates asymmetrically the coupling between the molecule and the leads (see Fig. 11). The rest of the paper is organized as follows: in next section we present the model and obtain the Kondo Hamiltonians describing the low energy physics, in section III we first analyze the behavior of Kondo couplings and Kondo temperatures, then we present variational wave functions that allow to explore a wider range of parameters, in particular situations in which the phonon frequencies are of

*Deceased

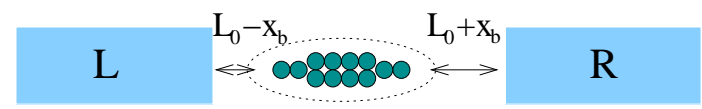

FIG. 1: Scheme of the molecular device studied in this work. A molecule is attached to contacts: $L$ and $R$, the center-ofmass motion modulates the molecule-leads tunneling barriers.

the order or smaller than the Kondo temperature. Section IV contains a study of the spectral density of the CMM phonon. Finally, last section summarizes results and conclusions.

\section{MODEL}

The model Hamiltonian is $H=H_{M}+H_{E}+H_{M E}$ where the first two terms describe the isolated molecule and electrodes, respectively, and the last term describes their coupling. We have

$$
\begin{aligned}
H_{M} & =\varepsilon_{d} n_{d}+U n_{d \uparrow} n_{d \downarrow}-\lambda\left(n_{d}-1\right)\left(a+a^{\dagger}\right) \\
& +\omega_{0} a^{\dagger} a+\omega_{1} b^{\dagger} b \\
H_{E} & =\sum_{k, \sigma, \alpha} \varepsilon_{\alpha k} c_{\alpha k \sigma}^{\dagger} c_{\alpha k \sigma} \\
H_{M E} & =\sum_{k, \sigma, \alpha} V_{\alpha}\left(x_{b}\right)\left(d_{\sigma}^{\dagger} c_{\alpha k \sigma}+c_{\alpha k \sigma}^{\dagger} d_{\sigma}\right)
\end{aligned}
$$

Here $n_{d}=n_{d \uparrow}+n_{d \downarrow}, n_{d \sigma}=d_{\sigma}^{\dagger} d_{\sigma}, d_{\sigma}^{\dagger}$ creates an electron at the molecular orbital with energy $\varepsilon_{d}, U$ is the intralevel Coulomb repulsion and $c_{\alpha k \sigma}^{\dagger}$ creates an electron in the $k$ mode of lead $\alpha=R, L$. The operators $a^{\dagger}$ and $b^{\dagger}$ create a phonon excitation on the Holstein mode with energy $\omega_{0}$ and on the CMM with energy $\omega_{1}$, respectively. The single particle energies are measured from the Fermi energy and $\hbar=1$.

From hereon we consider a symmetric molecule with identical $R$ and $L$ leads $\left(\varepsilon_{\alpha k} \equiv \varepsilon_{k}\right)$ and take $V_{R}\left(x_{b}\right)=$ 
$V_{0}+g x_{b}$ and $V_{L}\left(x_{b}\right)=V_{0}-g x_{b}$, where $x_{b}=$ $\left(1 / 2 M \omega_{1}\right)^{1 / 2}\left(b+b^{\dagger}\right)$ is the molecular center-of-mass coordinate. For a molecule with inversion symmetry, $x_{b}$ cannot couple linearly with the electron density.

It is convenient to rewrite the Hamiltonian in terms of symmetric and antisymmetric modes defined by $c_{S k \sigma}=$ $\left(c_{R k \sigma}+c_{L k \sigma}\right) / \sqrt{2}$ and $c_{A k \sigma}=\left(c_{R k \sigma}-c_{L k \sigma}\right) / \sqrt{2}$ :

$$
\begin{aligned}
H_{M E} & =V_{S} \sum_{k, \sigma}\left(d_{\sigma}^{\dagger} c_{S k \sigma}+c_{S k \sigma}^{\dagger} d_{\sigma}\right) \\
& +V_{A}\left(b+b^{\dagger}\right) \sum_{k, \sigma}\left(d_{\sigma}^{\dagger} c_{A k \sigma}+c_{A k \sigma}^{\dagger} d_{\sigma}\right)
\end{aligned}
$$

with $V_{S}=\sqrt{2} V_{0}$ and $V_{A}=\sqrt{1 / M \omega_{1}} g$. This shows that the symmetric mode is directly coupled to the molecular orbital while the antisymmetric coupling is phononassisted.

In the Kondo limit we eliminate the charge fluctuations by means of a Schrieffer-Wolff transformation extending the analysis of Refs. [11, 12,21]. We obtain that the spin fluctuations are described by an asymmetric two-channel Kondo Hamiltonian

$$
H_{K}=\sum_{\nu=A, S} J_{\nu} \sum_{k, k^{\prime}, \sigma, \sigma^{\prime}} \vec{S} \cdot c_{\nu k \sigma}^{\dagger} \frac{\vec{\sigma}_{\sigma \sigma^{\prime}}}{2} c_{\nu k^{\prime} \sigma^{\prime}}
$$

Here $\vec{S}$ is the spin operator of the molecular orbital, $\vec{\sigma}$ are the Pauli matrices, and

$$
\begin{aligned}
& J_{S}=\sum_{m=0}^{\infty}\left(\frac{2 V_{S}^{2} \gamma_{m}}{-\widetilde{\varepsilon}_{d}+m \omega_{0}}+\frac{2 V_{S}^{2} \gamma_{m}}{\widetilde{\varepsilon}_{d}+\widetilde{U}+m \omega_{0}}\right), \\
& J_{A}=\sum_{m=0}^{\infty}\left(\frac{2 V_{A}^{2} \gamma_{m}}{-\widetilde{\varepsilon}_{d}+\omega_{1}+m \omega_{0}}+\frac{2 V_{A}^{2} \gamma_{m}}{\widetilde{\varepsilon}_{d}+\widetilde{U}+\omega_{1}+m \omega_{0}}\right),
\end{aligned}
$$

where $\gamma_{m}=e^{-\left(\lambda / \omega_{0}\right)^{2}}\left(\lambda / \omega_{0}\right)^{2 m} / m !, \widetilde{\varepsilon}_{d}=\varepsilon_{d}+\lambda^{2} / \omega_{0}$, $\widetilde{U}=U-2 \lambda^{2} / \omega_{0}, \widetilde{\varepsilon}_{d}<0$, and $\widetilde{U}>0$. To second order in $H_{M E}$ there are also inelastic contributions proportional to $V_{S} V_{A}$ which couple the ground state of the isolated molecule to a molecular state with a CMM excitation. The energy level structure of the molecule is analogous to that of a multilevel quantum dot with level splitting $\omega_{1}$, and for large $\omega_{1}$ these contributions can be neglected. $\stackrel{22}{ }$ We will present below a more detailed calculation where this assumption is not required.

\section{SPIN SCREENING IN THE KONDO LIMIT}

The low energy properties of $H_{K}$ are well known $\underline{23}$ At low temperatures only one channel participates in the screening of the molecular spin, and the Fermi liquid picture applies as $T \rightarrow 0$. In the renormalization group language, the zero temperature fixed point corresponds to a diverging ratio of the largest to the smallest Kondo coupling. Only for $J_{S}=J_{A}$ both channels participate in

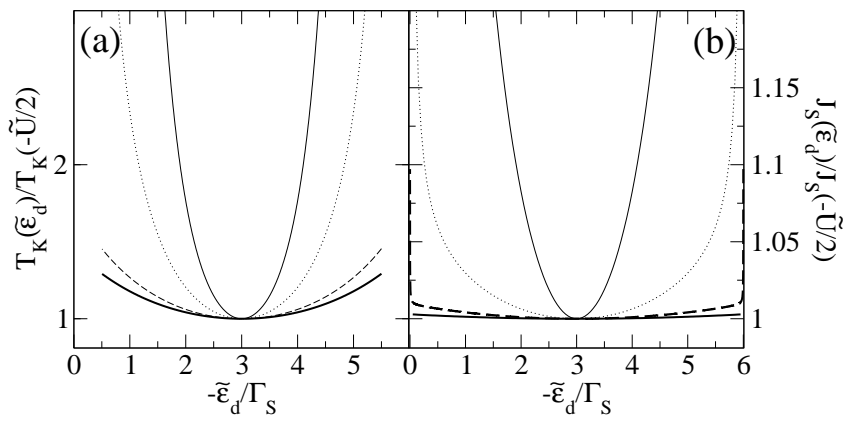

FIG. 2: a) The Kondo temperature plotted against $\widetilde{\varepsilon}_{d}$ for different values of the $e$-ph coupling $\lambda . \lambda / \omega_{0}=1$ (thin line), 2.5 (dotted line), 3.5 (dashed line), and 4.5 (thick line). Here $\Gamma_{S}=\pi \rho V_{S}^{2}=20 \mathrm{meV}, \widetilde{U}=6 \Gamma_{S}$, and $\omega_{0} / \widetilde{U}=0.5$. b) Kondo coupling $J_{S}$ against $\widetilde{\varepsilon}_{d}$ for the parameters of a).

an overscreening of the molecular spin. In our context, this would be a quite peculiar situation. In the moderate or weak $e$-ph coupling regime $V_{A}$ is smaller than $V_{S}$ and the Kondo coupling $J_{S}$ is therefore larger than $J_{A}$. The screening of the molecular spin is then dominated by the symmetric channel. In this case the Kondo temperature is given by $T_{K}=D e^{-1 / \rho J_{S}}$, where $D$ is a high energy cutoff and $\rho$ is the bare electronic density of states of the leads at the Fermi level. These results imply that, at zero temperature, the asymmetric channel is effectively decoupled from the molecule and the conductance is governed by the symmetric channel. As a consequence the conductance is simply $2 e^{2} / h$.

In what follows we show that the obtained $J_{S}$ may explain some experimentally observed anomalies on the gate voltage dependence of $T_{K} \stackrel{\underline{4}, 6}{ }$ In the absence of $e$ ph coupling $(\lambda=0) J_{S}$ is given by the usual expression $J_{S}(\lambda=0)=-2 V_{S}^{2} U /\left[\varepsilon_{d}\left(\varepsilon_{d}+U\right)\right]$ and increases as the gate voltage decreases $\left|\varepsilon_{d}\right|$ or $\left(\varepsilon_{d}+U\right)$. The experimentally observed dependence of the Kondo temperature on the gate voltage is, however, much weaker than what is inferred from this expression. In Ref. 6 the Kondo temperature remains almost independent of the gate voltage and presents a fast increase very close to the charge degeneracy points. Other systems also present a change in the Kondo temperature dependence on gate voltage at the borders of the Coulomb blockade valley $\underline{\underline{4}}$

These anomalies can be understood by the presence of $e$-ph coupling. Using the expression for $J_{S}$ from Eq. (6) we plotted the gate voltage dependence of the Kondo temperature for different values of the $e$-ph coupling (see Fig. 21). The gate voltage dependence of $T_{K}$ weakens monotonically with increasing $e$-ph interaction. This can be seen more easily making some simplifying approximations on Eq. (6) for $\widetilde{\varepsilon}_{d} \sim-\widetilde{U} / 2$. Note that the relevant quantities here are $\widetilde{\varepsilon}_{d}$ and $\widetilde{U}$ since the valence changes in the molecule occur for $\widetilde{\varepsilon}_{d}=0$ and $\widetilde{\varepsilon}_{d}=-\widetilde{U}$. One of the effects of the $e$-ph coupling is to decreases both $\left|\widetilde{\varepsilon}_{d}\right|$ and $\widetilde{U}$ shrinking the region of valence stability. For large $\lambda / \omega_{0} \gg 1, \gamma(m) \equiv \gamma_{m}$ is approximately Gaussian 
peaked at $m \sim m^{\star}=\left(\lambda / \omega_{0}\right)^{2}$ of width $\sqrt{m^{\star}}$ and satisfies $\sum_{m} \gamma_{m}=1$. In this case, the series in Eq. (6) can be approximated with

$$
\begin{aligned}
J_{S} & \simeq 2 V_{S}^{2}\left(\frac{1}{-\widetilde{\varepsilon}_{d}+m^{\star} \omega_{0}}+\frac{1}{\widetilde{U}+\widetilde{\varepsilon}_{d}+m^{\star} \omega_{0}}\right) \\
& \simeq 2 V_{S}^{2}\left(\frac{1}{-\varepsilon_{d}}+\frac{1}{U+\varepsilon_{d}}\right) .
\end{aligned}
$$

We obtain in the large $\lambda / \omega_{0}$ limit that $J_{S}$ is given by the $\lambda=0$ expression! This result, however, ceases to be valid close to the charge degeneracy points $\widetilde{\varepsilon}_{d} \rightarrow 0,-\widetilde{U}$ where the $m=0$ term in Eq. (6) diverges with an exponentially small Franck-Condon prefactor $\gamma_{0}=e^{-\left(\lambda / \omega_{0}\right)^{2}}$. The smallness of this prefactor requires $\widetilde{\varepsilon}_{d}$ or $\widetilde{\varepsilon}_{d}+\widetilde{U}$ to be exponentially small in order to make the $m=0$ term of order one [see Fig. 2(b)]. As a consequence, $J_{S}$ behaves as $J_{S}(\lambda=0)$ which is regular at the charge degeneracy points and only very close to them in behaves as $J_{S} \sim-2 V_{S}^{2} \widetilde{U} /\left[\widetilde{\varepsilon}_{d}\left(\widetilde{\varepsilon}_{d}+\widetilde{U}\right)\right]$, which is divergent. The latter expression gives the behavior of $J_{S}$ that can naively expected using the renormalized single particle energy $\widetilde{\varepsilon}_{d}$ and Hubbard interaction $\widetilde{U}$ in the usual expression for the Kondo coupling in an Anderson model. The behavior of the Franck-Condon matrix elements $\gamma_{m}$ is the source of the anomalous dependence of the Kondo temperature on the gate voltage. Related anomalies are obtained in the valence change of simpler non-interacting models $\frac{9}{-}$ and in the incoherent non-linear transport $\underline{18}$

It is important to notice that to lowest order in the hybridization only the Holstein coupling renormalizes $J_{S}$ and produces the anomalous behavior. In what follows we show that in higher order the CMM contributes to increase the Kondo coupling an effect that may help explain the high values of $T_{K}$ observed in some devices. To do this we now return to the original description given by the full Hamiltonian $H$ and build the ground state wavefunction using a generalized Varma-Yafet ansatz $\stackrel{\underline{24}}{=}$ While this method is exact only in the large $N$ limit, where the spin symmetry is extended to the $S U(N)$ group, it gives the correct zero-temperature physics even in the spin $1 / 2$ $(N=2)$ case. Following Ref $\frac{25}{2}$ we propose for the ground state wavefunction:

$$
|\Phi\rangle=b_{0}\left|\phi_{F}\right\rangle\left|P_{0}\right\rangle+\sum_{k, \sigma, \nu=A, S} b_{\nu k}\left|\phi_{\nu k}\right\rangle\left|P_{\nu k}\right\rangle,
$$

where $\left|\phi_{F}\right\rangle$ describes the Fermi sea with total energy $E_{F S},\left|\phi_{\nu k}\right\rangle=\frac{1}{\sqrt{2}} \sum_{\sigma} d_{\sigma}^{\dagger} c_{\nu k \sigma}\left|\phi_{F}\right\rangle,\left|P_{0}\right\rangle$, and $\left|P_{\nu k}\right\rangle$ are phonon states including both the Holstein coupling and the CMM, and we considered the $U \rightarrow \infty$ limit. The coefficients $b_{0}$ and $b_{\nu k}$ are chosen for the wavefunction to satisfy the Schrödinger equation $H|\Phi\rangle=E_{G S}|\Phi\rangle$.

A simple procedure allows to determine the form of the phonon states and their coefficients. First we note that $\left\langle\phi_{F}|H| \Phi\right\rangle=E_{G S} b_{0}\left|P_{0}\right\rangle$ and $\left\langle\phi_{\nu k}|H| \Phi\right\rangle=$ $E_{G S} b_{\nu k}\left|P_{\nu k}\right\rangle$. This generates a system of coupled equations for the phonon states that, after eliminating $b_{\nu k}\left|P_{\nu k}\right\rangle$, can be cast in the form

$$
\begin{aligned}
\left(\delta E+\varepsilon_{d}\right)\left|P_{0}\right\rangle & =\left[H_{M}^{0}+2 V_{S}^{2} \Gamma(\widehat{E})\right. \\
& \left.+2 V_{A}^{2}\left(b+b^{\dagger}\right) \Gamma(\widehat{E})\left(b+b^{\dagger}\right)\right]\left|P_{0}\right\rangle
\end{aligned}
$$

Here $\delta E=E_{G S}-E_{F S}-\varepsilon_{d}$ is the energy gained due to the hybridization, $\widehat{E}=-\delta E-\varepsilon_{d}+H_{M}^{1}, H_{M}^{\ell}$ is the projection of $H_{M}$ on the subspace with $\ell$ electrons in the molecular orbital, and

$$
\Gamma(z)=\sum_{k \leq k_{F}} \frac{1}{\varepsilon_{k}-z}=\frac{1}{2 D} \ln \left|\frac{z}{D+z}\right|,
$$

where we considered for simplicity a square half-filled conduction band of total width $2 D$. We will study first the case with $\lambda=0$. In this case the state $\left|P_{0}\right\rangle$ describes only the CMM degrees of freedom and can be expanded in the base of eigenstates of the uncoupled phonon $\left|P_{0}\right\rangle=\sum_{n} \alpha_{n}|n\rangle$. The following recursion relation is obtained for the coefficients:

$$
M_{n n} \alpha_{n}=M_{n, n+2} \alpha_{n+2}+M_{n, n-2} \alpha_{n-2},
$$

where

$$
\begin{aligned}
M_{n, n} & =\delta E+\varepsilon_{d}-\omega_{1} n-2 V_{S}^{2} \Gamma_{n} \\
& -2 V_{A}^{2}\left[(n+1) \Gamma_{n+1}+n \Gamma_{n-1}\right], \\
M_{n, n+2} & =2 V_{A}^{2} \sqrt{(n+1)(n+2)} \Gamma_{n+1}, \\
M_{n, n-2} & =2 V_{A}^{2} \sqrt{n(n-1)} \Gamma_{n-1},
\end{aligned}
$$

with $\Gamma_{n}=\Gamma\left(-\delta E+\omega_{1} n\right)$. The phonon state $\left|P_{0}\right\rangle$ can only include even or odd $n$ states and therefore has a well defined parity. We can find approximate solutions of these equations by noting that in the Kondo limit $\delta E$ is small. We have

$$
\Gamma_{0} \simeq \frac{1}{2 D} \ln \left|\frac{\delta E}{D}\right| \text { and } \Gamma_{n} \simeq \frac{1}{2 D} \ln \left|\frac{\omega_{1} n}{D+\omega_{1} n}\right| \text { for } n>0,
$$

that give for the even- $\left|P_{0}\right\rangle$ subspace

$$
\left(\delta E+\varepsilon_{d}-2 V_{S}^{2} \Gamma_{0}-2 V_{A}^{2} \Gamma_{1}-\Sigma_{S}\right) \alpha_{0}=0,
$$

where $\Sigma_{S}$ is a small correction that can be written as a continued fraction after eliminating all coefficients $\alpha_{n}$ with $n>0$. Equation (15) gives a simple self-consistent condition for $\delta E$ and in the weak coupling regime the usual solution is obtained

$$
\delta E=-D e^{-\frac{1}{\rho J_{S}^{\prime}}},
$$

where $\rho=(2 D)^{-1}$ and

$$
J_{S}^{\prime}=\frac{2 V_{S}^{2}}{\left|\varepsilon_{d}\right|+2 V_{A}^{2} \Gamma_{1}+\Sigma_{S}} .
$$

The energy gain $\delta E$ is associated to the formation of the Kondo singlet and is of the order of the Kondo temperature. This result shows that, in the even- $\left|P_{0}\right\rangle$ subspace, 
the molecular spin is Kondo screened by the symmetric channel, and the antisymmetric one only contributes to a renormalization of the molecular energy. This is the ground state for $V_{S} \gtrsim V_{A}$. In the opposite situation the ground state belongs to the odd- $\left|P_{0}\right\rangle$ subspace, and the antisymmetric channel screens the molecular spin with a Kondo coupling constant

$$
J_{A}^{\prime}=\frac{2 V_{A}^{2}}{\left|\varepsilon_{d}\right|+2 V_{S}^{2} \Gamma_{1}+2 V_{A}^{2} \Gamma_{2}+\Sigma_{A}+\omega_{1}} .
$$

Except for the presence of molecular energy correction terms in the denominators, $J_{S}^{\prime}$ and $J_{A}^{\prime}$ reproduce the Kondo couplings of Eq. (6) particularized for $\lambda=0$ and $U \rightarrow \infty$. The same procedure can be followed when both $e$-ph couplings are non-zero and the result of Eq. (6) recovered. This analysis not only validates the results of Eq. (5) and Eq. (6) for $\omega_{0}, \omega_{1} \gg T_{K}$ but it also gives some insight on the physics when $\omega_{0}$ or $\omega_{1}$ are of the order or smaller than $T_{K}$. To illustrate this we consider $\lambda=0$ and $\omega_{1} \gtrsim T_{K}$. The dependence of $\Gamma_{1}$ on $\delta E$ [see Eqs. (13)] now needs to be considered and the resulting equation for $\delta E$ can be solved numerically. The main result is an increasing $|\delta E|$ (an increasing Kondo temperature) with decreasing $\omega_{1}$, an effect that may be related to the high Kondo temperatures observed in some devices. 6

\section{PHONON SPECTRAL DENSITY}

Having characterized the nature of the Kondo screening, we now analyze the molecular vibrational modes. We define the phonon propagators in terms of the phonon fields $\phi_{0}=a+a^{\dagger}$ and $\phi_{1}=b+b^{\dagger}$

$$
\mathcal{D}_{\eta}(t) \equiv\left\langle\left\langle\phi_{\eta}(t), \phi_{\eta}(0)\right\rangle\right\rangle=-i \theta(t)\left\langle\left[\phi_{\eta}(t), \phi_{\eta}(0)\right]\right\rangle
$$

for $\eta=0,1$. Calculating the self-energy to second order in the $e$-ph interaction we obtain

$$
\mathcal{D}_{\eta}(\omega)=\frac{2 \omega_{\eta}}{\omega^{2}-\omega_{\eta}^{2}-2 \omega_{\eta} \Pi_{\eta}(\omega)}
$$

where $\mathcal{D}_{\eta}(\omega)$ indicates the Fourier transform of $\mathcal{D}_{\eta}(t)$. This quantity has been studied by Hewson et al ${ }^{26}$ for a Holstein coupling, where the self-energy $\Pi_{0}(\omega)$ is given by the charge susceptibility. In the Kondo limit (for $\widetilde{U}>0$ ) it has little structure at low frequency and the vibrational mode just acquires a small width due to the coupling with the electrons at the molecular orbital. The self-energy for the CMM is given by

$$
\begin{aligned}
\Pi_{1}(\omega) & =2 V_{A}^{2} \sum_{k, \sigma}\left(\left\langle\left\langle d_{\sigma}^{\dagger} c_{A k \sigma}, c_{A k \sigma}^{\dagger} d_{\sigma}\right\rangle\right\rangle_{\omega}\right. \\
& \left.+\left\langle\left\langle c_{A k \sigma}^{\dagger} d_{\sigma}, d_{\sigma}^{\dagger} c_{A k \sigma}\right\rangle\right\rangle_{\omega}\right) .
\end{aligned}
$$

Since the Kondo screening is due to the symmetric channel, the antisymmetric electronic modes can be decoupled from the molecular orbital and $\Pi_{1}(\omega)$ calculated in the
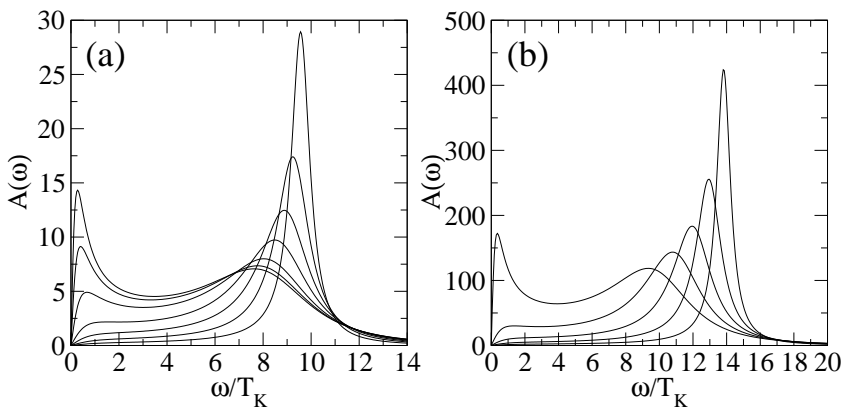

FIG. 3: Phonon spectral density $\mathcal{A}(\omega)$. a) $\omega_{1}=0.25 D$, $\omega_{1} / T_{K}=10$, and $\varsigma / T_{K}=0.15,0.25,0.35,0.45,0.55,0.6$, and 0.625 (from bottom to top in the low energy region). b) $\omega_{1}=$ $0.025 D, \omega_{1} / T_{K}=15, \varsigma / T_{K}=0.15,0.25,0.35,0.45$, and 0.55 (from bottom to top in the low energy region).

lowest order in $V_{A} . \Pi_{1}(\omega)$ can then be written in terms of one-particle propagators that for $T \ll T_{K}$ and $\omega \ll\left|\varepsilon_{d}\right|$ are given by $-\frac{1}{\pi} \sum_{k} \operatorname{Im}\left\langle\left\langle c_{A k \sigma}, c_{A k \sigma}^{\dagger}\right\rangle\right\rangle_{\omega+i 0}=\rho$ and

$$
-\frac{1}{\pi} \operatorname{Im}\left\langle\left\langle d_{\sigma}, d_{\sigma}^{\dagger}\right\rangle\right\rangle_{\omega+i 0}=\frac{T_{K}}{\Gamma_{S}} \frac{T_{K} / \pi}{\omega^{2}+T_{K}^{2}} .
$$

We have

$$
\begin{aligned}
\Pi_{1}(\omega) & =2 V_{A}^{2} \rho \frac{T_{K}}{\Delta} \int d \nu_{1} \int d \nu_{2}\left[\frac{f\left(\nu_{1}\right)-f\left(\nu_{2}\right)}{\omega+\nu_{1}-\nu_{2}+i 0}\right. \\
& \left.+\frac{f\left(\nu_{2}\right)-f\left(\nu_{1}\right)}{\omega-\nu_{1}+\nu_{2}+i 0}\right] \frac{T_{K} / \pi}{\nu_{1}^{2}+T_{K}^{2}} .
\end{aligned}
$$

At zero temperature the integrals reduce to a simple form and the phonon propagator reads:

$$
\mathcal{D}_{1}(\omega)=\frac{2 \omega_{1}}{\omega^{2}-\omega_{1}^{2}-2 \omega_{1} \varsigma\left[\ln \left(\frac{\omega^{2}+T_{K}^{2}}{D^{2}}\right)-i 2 \arctan \left(\frac{\omega}{T_{K}}\right)\right]}
$$

with $\varsigma=2 V_{A}^{2} \rho T_{K} / \Gamma_{S}=\left(V_{A} / V_{S}\right)^{2} 2 T_{K} / \pi$.

The phonon spectral density $\mathcal{A}(\omega)=-\operatorname{Im} \mathcal{D}_{1}(\omega) / \pi$ is shown in Fig. 3 for different values of the microscopic parameters. For small $e$-ph coupling it presents a single peak at $\sim \omega_{1}$ broadened by the coupling to the electronic degrees of freedom. For temperatures lower than $T_{K}$ and moderate $e$-ph couplings, the system presents a soft mode (low energy peak). For temperatures larger than $T_{K}$ (not shown) the soft mode disappears as the low-frequency spectral density of the $d$-electrons is structureless and small.

The emergence of the soft mode is associated to the onset of a dynamical Jahn-Teller distortion ${ }^{25}$ induced by Kondo correlations. To illustrate this we perform a semiclassical treatment in which the CMM displacement $x_{b}$ is replaced by a real number. For simplicity we will consider only the case $\lambda=0$, but the conclusions are valid in the general case. The molecule is coupled to the left and right leads with hopping amplitudes $V_{L}=\left(V_{0}+g x_{b}\right)$ and $V_{R}=\left(V_{0}-g x_{b}\right)$, respectively. For a given value of the 
C-number $x_{b}$ we can perform a unitary transformation

$$
\left(\begin{array}{c}
c_{1 k \sigma} \\
c_{2 k \sigma}
\end{array}\right)=\frac{1}{\sqrt{V_{L}^{2}+V_{R}^{2}}}\left(\begin{array}{cc}
V_{L} & V_{R} \\
-V_{R} & V_{L}
\end{array}\right)\left(\begin{array}{c}
c_{L k \sigma} \\
c_{R k \sigma}
\end{array}\right) .
$$

to a new base where the $\left\{c_{2 k \sigma}\right\}$ are decoupled from the molecule and play no role in the Kondo physics while the $\left\{c_{1 k \sigma}\right\}$ are coupled with a tunneling amplitude $\sqrt{2} V_{0} \sqrt{1+\frac{g^{2}}{V_{0}^{2}} x_{b}^{2}}$ that determines the Kondo screening. The Kondo temperature for this semiclassical treatment is therefore given by

$$
T_{K}^{C}=D e^{-1 /\left[\rho J_{S}\left(1+\frac{g^{2}}{V_{0}^{2}} x_{b}^{2}\right)\right]} .
$$

We can write the zero-temperaure energy gain of the system as a sum of the electronic [see Eq. (16)] and phononic contributions ${ }^{25,27}$

$$
E\left(x^{\prime}\right)=-D e^{-1 /\left[\rho J_{S}\left(1+x^{\prime 2}\right)\right]}+\frac{1}{2 \pi} \omega_{1} \frac{T_{K}}{\varsigma} x^{\prime 2},
$$

where we have introduced the reduced displacement $x^{\prime}=$ $\frac{g}{V_{0}} x_{b}$. In Fig. 4 we plot the energy gain as a function of $x^{\prime}$ for the same parameters as in Fig. 3 $\mathrm{k}$. For small $e$-ph coupling, $\varsigma / T_{K} \ll 1$, the energy has a parabolic form and the minimum is for $x^{\prime}=0$. The curvature of the parabola decreases with increasing $\varsigma$ and this is consistent with the shift to lower energies of the main peak in the spectral density (see Fig. [3 $\mathrm{a}$ ). For $\varsigma / T_{K} \gtrsim 0.3$ two symmetric minima emerge for non-zero values of $x^{\prime}$ showing that there is there is a Jahn-Teller instability associated with the formation of the Kondo effect. This Jahn-Teller distortion has associated a soft mode which is reflected in the phonon spectral density as a low energy peak.

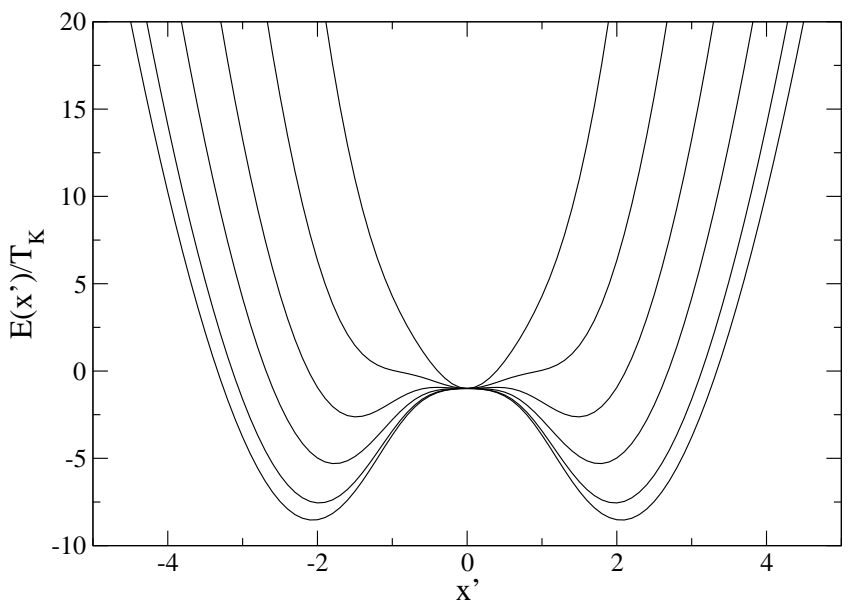

FIG. 4: Energy gain as a function of the reduced displacement $x^{\prime}=\frac{g}{V_{0}} x_{b}$ of the CMM for different values of the electron phonon coupling $\varsigma / T_{K}=0.15,0.25,0.35,0.45,0.55,0.6$, and 0.625 (from top to bottom). Other parameters as in Fig. [3

\section{SUMMARY AND CONCLUSIONS}

We have studied analytically electron-phonon correlation effects in molecular transistors using SchriefferWolff transformations and a Varma-Yafet ansatz. While the first technique provides a description in terms of low energy Hamiltonians, the latter allows the study of the whole range of parameters, including situations in which the phonon frequencies are smaller than the Kondo temperature. We considered a Holstein coupling and a center-of-mass mode and showed that both produce a renormalization of the Kondo temperature. Remarkably, the Holstein coupling renormalizes the Kondo coupling and weakens the gate voltage dependence of $T_{K}$. This may explain the puzzling behavior observed in molecular $\mathrm{Co}$ and $\mathrm{Cu}$ complexes .4 .6

The center-of-mass motion of the molecule opens an additional channel for charge and spin fluctuations. At low energies the latter are described by an asymmetric two-channel Kondo Hamiltonian. Due to channel asymmetry, however, the screening of the localized spin will be in general dominated by a single channel. While the temperature dependence of the conductance may present some anomalies due to the additional channel, at low temperatures the system is a Fermi liquid and the low bias conductance through the molecule is $G \sim 2 e^{2} / h$. Our calculations show that, in the Kondo regime a single channel is active at zero temperature and no interference is possible between direct and phonon assisted channels $\stackrel{30}{ }$.

Finally, we showed that the phonon spectral density of the center-of-mass mode presents a low energy structure induced by the coupling to the Kondo quasiparticles. Recent technical advances in single molecule phonon spectroscopy ${ }^{28,29}$ may allow for a direct study of this effect and provide useful information on the $e$-ph interaction in these systems.
1 A. Nitzan and M. A. Ratner, Science 300, 1384 (2003).

2 J. Park, A. N. Pasupathy, J. I. Goldsmith, C. Chang, Y. Yaish, J. R. Petta, M. Rinkoski, J. P. Sethna, H. D.
Abruña, P. L. McEuen, et al., Nature 417, 722 (2002).

3 S. Kubatkin, A. Danilov, M. Hjort, J. Cornil, J.-L. Brédas, N. Stuhr-Hansen, P. Hedegård, and T. Bjørnholm, Nature 
425, 698 (2003)

${ }^{4}$ W. Liang, M. P. Shores, M. Bockrath, J. R. Long, and H. Park, Nature 417, 725 (2002).

5 L. H. Yu and D. Natelson, Nano Letters 4(1), 79 (2004).

${ }^{6}$ L. H. Yu, Z. K. Keane, J. W. Ciszek, L. Cheng, J. M. Tour, T. Baruah, M. R. Pederson, and D. Natelson, Phys. Rev. Lett. 95, 256803 (2005).

7 W. Ho, J. Chem. Phys. 55, 11033 (2002).

${ }^{8}$ H. Park, J. Park, A. K. L. Lim, E. H. Anderson, A. P. Alivisatos, and P. L. McEuen, Nature 407, 57 (2000).

9 S. Braig and K. Flensberg, Phys. Rev. B 68, 205324 (2003).

10 A. Mitra, I. Aleiner, and A. J. Millis, Phys. Rev. B 69, 245302 (2004).

11 P. S. Cornaglia, H. Ness, and D. R. Grempel, Phys. Rev. Lett. 93, 147201 (2004).

12 P. S. Cornaglia, D. R. Grempel, and H. Ness, Phys. Rev. B 71, 075320 (2005).

13 P. S. Cornaglia and D. R. Grempel, Phys. Rev. B 71, 245326 (2005).

14 J. Paaske and K. Flensberg, Phys. Rev. Lett. 94, 176801 (2005).

15 L. Arrachea and M. J. Rozenberg, Phys. Rev. B 72, 041301(R) (2005).

16 J. Mravlje, A. Ramsak, and T. Rejec, Phys. Rev. B 72, 121403(R) (2005).

17 K. A. Al-Hassanieh, C. A. Busser, G. B. Martins, and E. Dagotto, Phys. Rev. Lett. 95, 256807 (2005).
18 J. Koch and F. von Oppen, Phys. Rev. Lett. 94, 206804 (2005).

19 J. Koch, M. E. Raikh, and F. von Oppen, Phys. Rev. Lett. 96, 056803 (2006).

${ }^{20}$ L. Y. Gorelik, A. Isacsson, M. V. Voinova, B. Kasemo, R. I. Shekhter, and M. Jonson, Phys. Rev. Lett. 80, 4526 (1998).

21 W. Stephan, M. Capone, M. Grilli, and C. Castellani, Phys. Lett. A 227, 120 (1997).

22 D. Boese, W. Hofstetter, and H. Schoeller, Phys. Rev. B 66, 125315 (2002).

23 P. Nozières and A. Blandin, J. Physique 41, 193 (1980).

24 C. M. Varma and Y. Yafet, Phys. Rev. B 13, 2950 (1976).

25 B. Alascio, C. Balseiro, G. Ortíz, M. Kiwi, and M. Lagos, Phys. Rev. B 38, 4698 (1988).

26 A. C. Hewson and D. Meyer, J. Phys.:Condens. Matter 14, 427 (2002).

27 D. P. Clougherty, Phys. Rev. Lett. 90, 035507 (2003).

${ }^{28}$ N. Hayazawa, A. Tarun, Y. Inouye, and S. Kawata, J. Appl. Phys. 92, 6983 (2002).

29 A. Hartschuh, E. J. Sanchez, X. S. Xie, and L. Novotny, Phys. Rev. Lett. 90, 095503 (2003).

30 Such interference was proposed in Ref. 17] where the authors considered a single phonon mode that modulates both the molecular level and the molecule-leads coupling. 